\title{
Molecular Sieving of Albumin by the Ascending Vasa Recta Wall
}

\author{
Thomas L. Pallone \\ Division of Nephrology, The M. S. Hershey Medical Center of the Pennsylvania State University, Hershey, Pennsylvania 17033
}

\begin{abstract}
Molecular sieving of albumin by ascending vasa recta. Evidence exists to support the presence of an extravascular pool of albumin in the renal medullary interstitium. This study used microperfusion in vivo to measure the transport of ${ }^{125}$ I-labeled albumin from descending (DVR) and ascending vasa recta (AVR) to the papillary interstitium. Perfusions were performed during furosemide diuresis with a buffer containing FITC-labeled dextran (FITC-Dx) $2 \times 10^{6} \mathrm{~mol}$ wt and ${ }^{125} \mathrm{I}$-albumin. Perfusate albumin and collection pressure were adjusted to induce either zero transcapillary volume flux (Jv) or high volume flux. When Jv was zero, the collectate-to-perfusate ratios of FITC-Dx $\left(R_{\mathrm{Dx}}\right)$ and ${ }^{125} \mathrm{I}$-albumin $\left(R_{\text {alb }}\right)$ in the DVR and AVR were identical implying that diffusive efflux of albumin was immeasurably small. In contrast, when $\mathrm{Jv}$ was increased, paired comparison of $R_{\text {alb }}$ and $R_{b x}$ in the same AVR revealed a difference, $1.58 \pm 0.06$ vs $1.72 \pm 0.08$, respectively $(P$ $<0.01)$. AVR perfusions in hydropenic animals showed similar results, $R_{\mathrm{alb}}=1.70 \pm 0.07$ and $R_{\mathrm{px}}=2.00 \pm 0.07(P<0.01)$. These data suggest that albumin transport across vasa recta in vivo is likely to be governed by solvent drag. The reflection coefficient of the AVR wall to ${ }^{125}$ I-albumin is estimated to be 0.78. (J. Clin. Invest. 1992. 90:30-34.) Key words: microcirculation • micropuncture $\bullet$ microperfusion $\bullet$ urinary concentration • rat
\end{abstract}

\section{Introduction}

In 1958, Lassen, Longley, and Lilienfield reported that autoradiograms of coronal sections taken from the kidneys of dogs injected with ${ }^{131} \mathrm{I}$-albumin showed intense medullary labeling. Similar experiments were also performed in which ${ }^{51} \mathrm{Cr}$-labeled red blood cells and iodinated albumin were injected and the renal vasculature flushed or ligated $(1,2)$. Those investigations suggested the existence of a large extravascular pool of albumin in the renal medulla. Injection of albumin labeled with various markers including radioiodine (3), FITC $(4,5)$, and Evans blue dye $(5,6)$ supported the presence of interstitial albumin. Histochemical methods have been used to examine the pathway by which macromolecules gain access to the interstitium. Tracers as large as catalase $(240,000 \mathrm{~mol} \mathrm{wt})$ and ferritin $(500,000 \mathrm{~mol} \mathrm{wt})$ crossed the fenestrations of cortical peritubular capillaries and ascending vasa recta $(\operatorname{AVR})^{1}(7,8)$.

Address correspondence and reprint requests to Thomas L. Pallone, M.D., Division of Nephrology, The Milton S. Hershey Medical Center, The Pennsylvania State University, P.O. Box 850, Hershey, PA 17033.

Received for publication 10 December 1991 and in revised form 21 February 1992.

J. Clin. Invest.

(c) The American Society for Clinical Investigation, Inc.

0021-9738/92/07/0030/05 \$2.00

Volume 90, July 1992, 30-34
The functional implication of macromolecular leakage into the medullary interstitium is obscure. The fact that a marker crosses capillary fenestrations does not provide insight into the magnitude or even the direction of net protein flux in vivo. The evidence that macromolecules gain access to the medullary interstitium is provocative, raising interesting and important questions with regard to the control of thermodynamic driving forces responsible for the removal of solutes and water by the microcirculation. An essential consideration in the formulation of a descriptive model of such transport processes concerns the absence of lymphatics from the inner medulla ( 9 , 10). Without lymphatic drainage, any transport of albumin could lead to an elevation of interstitial oncotic pressure.

To determine whether the descending vasa recta (DVR) (continuous endothelium) or AVR (fenestrated endothelium) transport albumin (11), these vessels were perfused in vivo with a buffer containing ${ }^{125} \mathrm{I}$-labeled albumin. To assess the relative contributions of diffusion and convection to albumin transport, perfusions were performed under conditions of near zero volume flux or high volume flux. Diffusive efflux of albumin from perfused descending or ascending vasa recta was too small to detect, however, convective transport across the AVR wall was readily demonstrated.

\section{Methods}

Female Munich-Wistar rats weighing 70-120 g were obtained from Harlan Sprague Dawley, Inc., Indianapolis, IN. The animals were allowed free access to food and water until the time of the experiment. Anesthesia was obtained by an intraperitoneal injection of thiopental, $50 \mathrm{mg} / \mathrm{kg}$ body wt. The rats were placed on a heated table and body temperature maintained between 36 and $38^{\circ} \mathrm{C}$ as measured by rectal probe. For infusions and bolus injections, the jugular vein was cannulated with polyethylene- 50 tubing that had been tapered by pulling over a flame. A tracheostomy was performed, the left femoral artery cannulated for blood pressure monitoring, and a bladder catheter inserted to measure urine flow from the right kidney. The papilla of the left kidney was prepared for micropuncture as previously described $(12,13)$. After surgery, a 30-min equilibration period was permitted to elapse before microperfusion was performed.

Animals subjected to furosemide and saline diuresis received a bolus injection of $0.14 \mathrm{mg} / 100 \mathrm{~g}$ body wt of furosemide in a volume of $0.2 \mathrm{ml} / 100 \mathrm{~g}$ body wt of saline followed by $2.3 \mu \mathrm{g} / \mathrm{min}$ per $100 \mathrm{~g}$ body wt of furosemide in $60 \mu \mathrm{l} / \mathrm{min}$ per $100 \mathrm{~g}$ body wt of saline (12). Animals maintained in the hydropenic state received only $15 \mu \mathrm{l} / \mathrm{min}$ per $100 \mathrm{~g}$ body wt of saline. Blood pressure and urinary flow rate were determined with each experiment. Values observed were similar to those previously encountered $(12,13)$.

1. Abbreviations used in this paper: A, AVR, ascending vasa recta; D, DVR, descending vasa recta; FD, furosemide diuresis; FITC-Dx, FITC-labeled Dx; HD, hydropenia; HJv, high volume flux; Jv, transcapillary volume flux; $0 \mathrm{Jv}$, zero $\mathrm{Jv} ; \mathbf{R}_{\mathrm{alb}}$, collectate-to-perfusate ratio of ${ }^{125}$ I-albumin; $R_{D x}$, collectate-to-perfusate ratio of FITC-Dx. 


\section{Experimental design}

The purpose of these experiments was to measure the loss of ${ }^{125} \mathrm{I}$-albumin from the lumen of AVR or DVR to the papillary interstitium under conditions of near zero volume flux $(0 \mathrm{Jv})$ or high volume flux $(\mathrm{HJv})$. To accomplish this task, microvessel segments were isolated between two paraffin blocks and perfused with the desired buffer as previously described (14). Transcapillary volume flux ( Jv) can be controlled by altering intracapillary Starling forces during microperfusion. Due to the high hydraulic conductivity of AVR wall, Jv is very large when these vessels are perfused with $0.1 \mathrm{~g} / \mathrm{dl}$ albumin at collection pressures of $15 \mathrm{mmHg}$ (14) and near zero with $5.0 \mathrm{~g} / \mathrm{dl}$ albumin (15). Table I summarizes experimental groups and the Starling forces employed to control volume flux across isolated vessel segments. The number of animals and vessels studied is also given. To eliminate concern over variation in interstitial $\mathrm{NaCl}$ and urea concentrations and the potential effects of transcapillary gradients of these solutes, most perfusions were performed under conditions of furosemide and saline diuresis (FD) (12). In one group, hydropenic conditions (HD) were maintained.

\section{Microvessel isolation and perfusion}

Individual vasa recta were blocked with paraffin and punctured twice, once at the papillary tip for microperfusion and a second time near the base to collect samples for analysis. Vessels were only perfused if a length, free of branch points, could be easily traced along the papillary surface. A paraffin block was placed near the papillary base (AVR) or tip (DVR) and the vessel observed to assure that the red blood cells became completely stagnant. If a branch point existed upstream of the block blood flow continued unabated through the branch. Such hidden branch points were rarely identified. A second paraffin block was placed as far from the first as possible (14). All perfusions were performed at $7.5 \mathrm{nl} / \mathrm{min}$ using a nanoliter infusion pump calibrated before and after these experiments. To control intracapillary pressure during perfusion, the hydraulic pressure in the collection pipette lumen was adjusted (14). The distance from the perfusion to collection sites was measured with an ocular micrometer.

Groups $0 J v-F D / A$ and $0 J v-F D / D$ (zero volume flux-furosemide diuresis/ascending vasa recta or /descending vasa recta). As shown in Table I these perfusions were performed using $5.0 \mathrm{~g} /$ deciliter ( $\mathrm{dl}$ ) albumin in perfusate buffer (see below) with the collection pipette pressurized to $10 \mathrm{mmHg}$. The purpose of these experiments was to determine whether diffusion of ${ }^{125} \mathrm{I}$-albumin from vasa recta to the interstitium could be detected under conditions of zero volume flux.

Groups $H J v-F D / A$ and $H J v-F D / D$ (high volume flux-furosemide diuresis/ascending vasa recta or /descending vasa recta). These perfusions were performed with $0.1 \mathrm{~g} / \mathrm{dl}$ albumin at $15 \mathrm{mmHg}$ during furosemide diuresis to measure convective transport of ${ }^{125} \mathrm{I}$-albumin from the vasa recta lumen to the papillary interstitium under conditions of high volume flux.

Group $H J v-H D / A$ (high volume flux-hydropenia/ascending vasa recta). These perfusions were performed with $0.1 \mathrm{~g} / \mathrm{dl}$ albumin at 15 $\mathrm{mmHg}$ during hydropenia to measure ${ }^{125} \mathrm{I}$-albumin flux. This group was included to determine if the results observed with group HJv-FD/ A could be reproduced in the absence of furosemide.

Table I. Microperfusions Performed

\begin{tabular}{ccccr}
\hline Group & Vessel & Cp & Pc & Na/Nv \\
& & $g / d l$ & $m m H g$ & \\
0Jv-FD/A & AVR & 5.0 & 10 & $10 / 19$ \\
0Jv-FD/D & DVR & 5.0 & 10 & $7 / 10$ \\
HJv-FD/A & AVR & 0.1 & 15 & $10 / 21$ \\
HJv-FD/D & DVR & 0.1 & 15 & $8 / 14$ \\
HJv-HD/A & AVR & 0.1 & 15 & $10 / 22$
\end{tabular}

Abbreviations: $\mathrm{Cp}$, albumin concentration; $\mathrm{Pc}$, collection pressure; $\mathrm{Na} / \mathrm{Nv}$, number of animals/number of vessels.
Perfusate buffer. For perfusions performed during furosemide diuresis the following buffer was used: $\mathrm{NaCl} 161 \mathrm{mM} ; \mathrm{NaH}_{2} \mathrm{PO}_{4} 0.29$ $\mathrm{mM} ; \mathrm{Na}_{2} \mathrm{HPO}_{4} 1.71 \mathrm{mM} ; \mathrm{KCl} 9.0 \mathrm{mM} ; \mathrm{MgCl}_{2} 1.0 \mathrm{mM} ; \mathrm{CaCl}_{2} 1.0$ $\mathrm{mM}$; urea $50 \mathrm{mM}$; Hepes $5 \mathrm{mM}$, pH $7.4(12,13)$. For hydropenic perfusions, concentrations of $\mathrm{NaCl}$, urea, and potassium were based upon measurements of vasa recta plasma composition at the tip of hydropenic Munich-Wistar rats under identical experimental conditions $(12,15) . \mathrm{NaCl} 322 \mathrm{mM} ; \mathrm{KCl} 28 \mathrm{mM}$; urea $360 \mathrm{mM} ; \mathrm{NaH}_{2} \mathrm{PO}_{4}$ $0.29 \mathrm{mM} ; \mathrm{Na}_{2} \mathrm{HPO}_{4} 1.71 \mathrm{mM} ; \mathrm{CaCl}_{2} 1 \mathrm{mM} ; \mathrm{MgCl}_{2} 1 \mathrm{mM}$; Hepes 5 $\mathrm{mM}$; pH 7.4. ${ }^{125} \mathrm{I}$-albumin (see below) and FITC-labeled dextran (FITC-Dx ), $2 \times 10^{6} \mathrm{~mol} \mathrm{wt}, 5 \mathrm{mg} / \mathrm{ml}$ (Sigma Chemical Co., St. Louis, $\mathrm{MO})$, were added to the perfusate.

As in previous studies, FITC-Dx served as a marker for the determination of transcapillary volume flux (13-15). In this laboratory, batches of FITC-Dx are routinely subjected to gel chromatography to examine their size distribution. All $2 \times 10^{6}$ average molecular weight FITC-Dx species to date have eluted near the void of a Sepharose 4B column (Pharmacia Inc., Piscataway, NJ). The tight binding of FITC to the dextran molecule has also been verified (13). FITC-Dx and ${ }^{125} \mathrm{I}$-albumin markers were dialyzed at weekly intervals (see below).

Iodination of albumin. BSA (A7906; Sigma Chemical Co.) was labeled with ${ }^{125}$ I (New England Nuclear, Boston, MA) using Iodo-beads (Pierce Chemical Co., Rockford, IL). $140 \mu$ l of PBS containing 0.4 $\mathrm{g} / \mathrm{dl}$ BSA was combined with three beads and $2 \mathrm{mCi}$ of ${ }^{125} \mathrm{I}$. The mixture reacted for $15 \mathrm{~min}$ after which iodinated BSA was isolated from free ${ }^{125}$ I by eluting from a 2-ml Sephadex G25 column (Pharmacia Inc.) with perfusate buffer. The void fractions were dialyzed against the desired perfusate buffer. Binding of ${ }^{125}$ I to albumin was verified using equilibrium dialysis and gel chromatography. Exhaustive dialysis of perfusate buffer and reexamination of binding was performed at weekly intervals. The rate of leakage of ${ }^{125}$ I from albumin was too small to quantitate with accuracy. It was $<1 \%$ per week when examined by equilibrium dialysis. ${ }^{125}$ I-albumin was also subjected to gel chromatography on a $60-\mathrm{cm}$ Sephadex G200 column at weekly intervals. It always eluted as a single peak near the void fraction.

\section{Analytical methods}

Microanalysis of FITC-Dx. The method used to measure FITC-Dx in collectate and perfusate is similar to that previously described, with minor modifications (13). Samples of perfusate and collectate were placed into a siliconized petri dish under water-equilibrated mineral oil. $7.5 \mu \mathrm{l}$ of PBS was loaded into 50- $\mu 1$ microcaps (Drummond Scientific Co., Broomall, PA) that had been cut in half. Using volumetric constriction pipettes, 1-nl volumes of samples were dispensed into the PBS. The ends of the microcap were flame sealed and centrifuged back and forth for mixing. Fluorescence was measured using a photon counting microscope detection assembly (model D104B; Photon Technology International, South Brunswick, $\mathrm{NJ}$ ) with the photomultiplier tube charged to $950 \mathrm{~V}$. Background fluorescence was subtracted from that measured in the perfusate and collectate to determine the fluorescence, $C_{0}$ and $C_{1}$, respectively. The rate of volume efflux $(\mathrm{Qv}, \mathrm{nl} / \mathrm{min})$ from the capillary to the papillary interstitium was calculated from the change in concentration of FITC-Dx from perfusate to collectate and the perfusion rate, Qo.

$Q_{v}=Q_{o}\left\{1-\frac{C_{0}}{C_{1}}\right\}$

Transcapillary volume flux is expressed per capillary length (L), Jvl $(\mathrm{nl} /[\mathrm{min} \cdot \mathrm{mm}])($ determined as $\mathrm{Qv} / \mathrm{L})$. Alternatively, volume flux can be given per unit of vessel wall surface area, $\mathrm{Jv}$ ( see below).

To measure ${ }^{125} \mathrm{I}$-albumin, the same cuvettes used to measure fluorescence were counted on a gamma counter (model 5500; Beckman Instruments, Fullerton, CA). Counts exceeded background by a factor of $8-10$. The protein assay was performed by the microadaptation of the Lowry method $(12,16)$.

Using these assays, the coefficient of variation for measurement of nine pipetted $1-n l$ samples of FITC-Dx and ${ }^{125}$ I-albumin was 0.045 and 0.039 , respectively. 
Theoretical analysis. The following demonstrates that the design of the $\mathrm{HJv}$ experiments provides a particularly powerful means for determining the sieving (or reflection) coefficient of the capillary wall to albumin.

Since the oncotic pressure of $0.1 \mathrm{~g} / \mathrm{dl}$ albumin is nearly zero and hydraulic pressure is expected to vary little along the perfused capillary (14), it can be assumed that $\mathrm{Jv}$ is independent of axial location.

$J_{v}=\frac{Q_{v}}{\Pi \mathrm{IDL}}$

where $\mathrm{Jv}$ is transcapillary volume flux expressed per unit of surface area $(\mathrm{cm} / \mathrm{s}), \mathrm{D}$ and $\mathrm{L}$ are the diameter and length of the perfused capillary, respectively. The flow rate $(\mathrm{Q})$ at any position $(\mathrm{x})$ is given by

$Q=Q_{o}-Q_{v}\left(\frac{\mathrm{x}}{\mathrm{L}}\right)$

Transcapillary albumin flux ( $\mathrm{Ja})$ is described by the equation of Patlak (17).

$J_{a}=J_{v} \Theta \frac{\mathrm{C}_{\mathrm{a}}-\mathrm{C}_{\mathrm{ai}} \mathrm{e}^{-\beta}}{1-\mathrm{e}^{-\beta}}$

$\Theta=1-\sigma_{\mathrm{a}} \quad \beta=\frac{\mathrm{J}_{\mathrm{v}} \Theta}{\mathrm{P}_{\mathrm{a}}}$

$\mathrm{C}_{\mathrm{a}}$ and $\mathrm{C}_{\mathrm{ai}}$ are the ${ }^{125} \mathrm{I}$ albumin concentration in the lumen and interstitium, respectively. $\sigma_{\mathrm{a}}$ and $\mathrm{Pa}$ are the reflection coefficient and permeability of the vasa recta wall to albumin.

Conservation of albumin requires

$\frac{d}{d x}\left(C_{a} Q\right)=-J_{a} \Pi D$

If $\beta$ in Eq. 4 is large, exponential terms approach zero and convection dominates as the mode of albumin transport. In that case, Eq. 4 becomes

$\mathbf{J}_{\mathrm{a}}=\mathbf{C}_{\mathbf{a}} \mathbf{J}_{\mathbf{v}} \boldsymbol{\theta}$

To justify the transition from Eq. 4 to Eq. 7 it is reasonable to require that the exponentials in the numerator and denominator of Eq. 4 to be $\ll 0.05$ or $\beta \gg 3$. Assuming the high volume flux perfusions satisfy this criterion, Eqs. 2, 3, 6, and 7 can be combined and integrated to yield

$\frac{C_{a}}{C_{a o}}=e^{\sigma_{2} \ln \left\{Q_{o} /\left[Q_{0}-Q_{v}\right]\right\}}$

Note that $\mathrm{Ca} / \mathrm{Cao}$ and $\mathrm{Qo} /(\mathrm{Qo}-\mathrm{Qv})$ are equal to the collectate-toperfusate ratio of ${ }^{125} \mathrm{I}$-albumin $\left(R_{\mathrm{alb}}\right)$ and FITC-Dx $\left(R_{\mathrm{Dx}}\right)$, respectively, so that a theoretical plot of $\mathbf{R}_{\mathrm{alb}}$ vs $\mathbf{R}_{\mathrm{bx}}$ should appear as shown in Fig. 1. Eq. 8 can be rearranged

$\ln \left(R_{a l b}\right)=\sigma_{\mathrm{a}} \ln \left(R_{D x}\right)$

A plot of the natural logarithm of $R_{a l b}$ vs $R_{b x}$ is expected to be linear, with a slope of $\sigma_{\mathrm{a}}$ and intercept at the origin. The power of Eq. 9 is apparent. $\sigma_{\mathrm{a}}$ can be determined from the collectate-to-perfusate marker ratios alone, without concern over the pericapillary interstitial ${ }^{125} \mathrm{I}$-al-

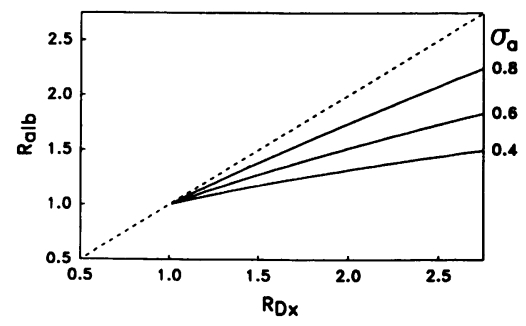

Figure 1. Theoretical results of single capillary perfusions for high volume flux conditions. The family of curves is calculated from Eq. 8 for values of $\sigma_{\mathrm{a}}=0.4$, 0.6 , or 0.8 . The graph depicts the collectate-to-perfusate

ratio of albumin ( $R_{\mathrm{alb}}$, ordinate) vs FITC-Dx $\left(\mathrm{R}_{\mathrm{Dx}}\right.$, abcissa). The dashed line is the line of identity, corresponding to $\sigma_{\mathrm{a}}=1$. bumin concentration or knowledge of vessel length and diameter. Furthermore, $\mathbf{R}_{\mathrm{Dx}}$ and $\mathbf{R}_{\mathrm{alb}}$ are measured in the same samples, eliminating pipetting as a source of error.

It follows that for Eq. 9 to be applied to analyze these experiments, rates of transcapillary volume flux must be sufficiently high for $\beta$ in Eq. 4 to be large. After presentation of the data, this issue will be examined in the Discussion section of this report.

Statistical analysis. Experimental results are reported as mean \pm SE. Statistical comparisons use paired or unpaired Student's $t$ tests as appropriate.

\section{Results}

$R_{\mathrm{Dx}}$ and $\mathrm{R}_{\mathrm{alb}}$ and the capillary segment lengths are shown for each group in Table II. For Groups 0Jv-FD/A and 0Jv-FD/D only, the collectate-to-perfusate ratio of albumin was determined for both ${ }^{125}$ I-albumin and for "chemical" albumin by the micro-Lowry method.

Transcapillary volume flux. The Starling forces chosen for the HJv groups ( Table I, collection pressure $=15 \mathrm{mmHg}$, albumin concentration $0.1 \mathrm{~g} / \mathrm{dl}$ ) successfully induced a high rate of transcapillary volume flux across AVR (groups HJv-FD/A, HJv-HD/A) but not DVR (Group HJv-FD/D) (Fig. 2). This result corroborates the difference in hydraulic conductivities previously observed for these two vessel types $(13,14)$. When perfusion was performed with $5.0 \mathrm{~g} / \mathrm{dl}$ albumin, $R_{\mathrm{Dx}}$ was indistinguishable from unity demonstrating that volume efflux was eliminated as intended for these groups (Table II, groups 0JvFD/A, 0Jv-FD/D).

Transcapillary albumin flux. No difference was found between the collectate-to-perfusate ratios of FITC-Dx and ${ }^{125} \mathrm{I}$-albumin or chemical albumin for the zero volume flux perfusions (Table II, groups OJv-FD/A, 0Jv-FD/D). This implies that diffusion of ${ }^{125} \mathrm{I}$-albumin from DVR or AVR lumen to papillary interstitium was undetectable by this experimental method. Similarly, $\mathbf{R}_{\mathrm{Dx}}$ and $\mathbf{R}_{\mathrm{alb}}$ were indistinguishable when volume flux across DVR segments was increased to $1.2 \mathrm{nl} /$ (min-mm) (Table II, HJv-FD/D). In contrast, in the AVR under conditions of high volume flux, ${ }^{125} \mathrm{I}$-albumin was concentrated to a lesser degree than FITC-Dx and paired comparison of $R_{b x}$ and $R_{a b b}$ revealed a highly significant difference between these markers (Table II, groups HJv-FD/A and HJvHD/A). This result demonstrates molecular sieving of ${ }^{125}$ I-albumin across the AVR wall during both furosemide diuresis and hydropenia. The results of individual perfusions are shown in the top and bottom panels of Fig. 3 where $R_{\text {alb }}$ is plotted as a function of $R_{D x}$ for all AVR perfusions. The deviation of points from the line of identity at higher values of $R_{D x}$ conforms to expectations based upon the theory provided in the Methods (Eq. 8 and Fig. 1).

Reflection coefficient of the AVR wall to albumin. In view of the similarity of the results obtained during high volume flux perfusions of the AVR during furosemide diuresis and hydropenia, the data from the 43 perfusions were combined and plotted logarithmically (Fig. 4). Consistent with theory, the regression line has an intercept that is statistically indistinguishable from zero (Methods, Eq. 9). The slope of the regression line in Fig. 4 provides an estimate of $\sigma_{\mathrm{a}}(0.78)$. When examined individually, a slope and intercept of 0.79 and 0.035 are obtained for the group HJv-FD/A, and 0.88 and -0.097 for group HJv-HD/A data, respectively. Combining the data in Fig. 4 to yield the best estimate of $\sigma_{\mathrm{a}}$ seems the best approach because these intercepts are not statistically different from zero and the slopes are not significantly different from one another. 
Table II. Collectate-to-Perfusate Marker Ratios

\begin{tabular}{|c|c|c|c|c|c|}
\hline Group & $R_{D x}$ & $\underset{\substack{R_{21 b} \\
\left({ }^{125} I\right)}}{ }$ & $\begin{array}{c}\mathrm{R}_{\mathrm{ab}} \\
\text { (Lowry) }\end{array}$ & Length & $\mathrm{Jvl}$ \\
\hline & & & & $\mu m$ & $n l /(\min -m m)$ \\
\hline 0Jv-FD/A & $0.98 \pm 0.03$ & $0.97 \pm 0.03$ & $0.97 \pm 0.04$ & $620 \pm 31$ & $-0.8 \pm 0.7$ \\
\hline $0 J v-F D / D$ & $1.02 \pm 0.03$ & $1.06 \pm 0.03$ & $1.01 \pm 0.02$ & $793 \pm 53$ & $-0.1 \pm 0.3$ \\
\hline $\mathrm{HJv}-\mathrm{FD} / \mathrm{A}$ & $1.72 \pm 0.08$ & $1.58 \pm 0.06^{*}$ & - & $666 \pm 47$ & $5.1 \pm 0.5$ \\
\hline HJv-FD/D & $1.13 \pm 0.04$ & $1.14 \pm 0.06$ & - & $728 \pm 39$ & $1.2 \pm 0.4$ \\
\hline HJv-HD/A & $2.00 \pm 0.07$ & $1.70 \pm 0.07^{*}$ & - & $666 \pm 35$ & $5.6 \pm 0.2$ \\
\hline
\end{tabular}

Entries are reported as mean \pm SE. ${ }^{*} P<0.01 \mathrm{R}_{\mathrm{alb}}$ vs $\mathrm{R}_{\mathrm{Dx}}$.

\section{Discussion}

A body of evidence suggests that macromolecules, particularly albumin, equilibrate between the microcirculation and renal medullary interstitium. The presence of a pool of extravascular albumin in the renal medulla has been confirmed by several studies (1-7). The present work was performed to examine the relative contributions of convection (solvent drag) and diffusion to albumin transport across the papillary vasa recta. The method used, free-flow microperfusion of single capillaries in vivo, has inherent limitations. First, if the rate of transport from lumen to interstitium is too low, a difference in mass flow rate between the point of perfusion and collection will not be measurable. Second, for albumin to escape from the capillary lumen, it must be consecutively transported across the capillary wall and through the pericapillary interstitium. Other investigators have examined the transport characteristics of the interstitium with respect to macromolecules $(18,19)$. Those studies demonstrate that as molecular weight rises, the interstitium provides an increasingly significant barrier to diffusion. The current experimental design overcomes the latter problem. Assuming that convection-dominated transport across the capillary wall has been achieved, analysis (Eqs. 3-9) with respect to the estimation of $\sigma_{\mathrm{a}}$ does not depend upon knowledge of interstitial ${ }^{125} \mathrm{I}$-albumin concentration.

Diffusive transport of albumin. An attempt was made to measure diffusion of albumin from lumen to interstitium. When DVR and AVR segments were perfused at $7.5 \mathrm{nl} / \mathrm{min}$ with buffer containing $5.0 \mathrm{~g} / \mathrm{dl}$ BSA at $10 \mathrm{mmHg}$, transcapillary volume flux was not significantly different from zero and efflux of ${ }^{125} \mathrm{I}$-albumin could not be detected (Table II, groups $0 \mathrm{Jv}-\mathrm{FD} / \mathrm{A}, 0 \mathrm{Jv}-\mathrm{FD} / \mathrm{D})$. If the capillary wall rather than pericapillary interstitium is the major barrier to diffusion, albumin permeability $(\mathrm{Pa})$ would be calculated from

$P_{a}=\frac{Q_{o}}{\Pi D L} \ln \left(\frac{1}{R_{\text {alb }}}\right)$

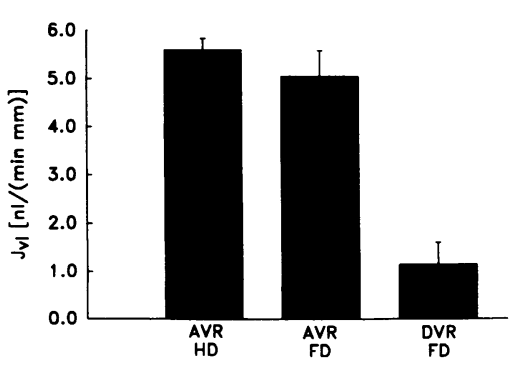

Figure 2. Transcapillary volume flux for AVR perfused during hydropenia (HD) or furosemide diuresis (FD) and DVR during furosemide diuresis. In all cases, vessels were perfused with buffer containing $0.1 \mathrm{~g} / \mathrm{dl}$ albumin at a collection pressure of $15 \mathrm{mmHg}$.
Assuming that a $5 \%$ change in ${ }^{125} \mathrm{I}$-albumin concentration $\left(R_{\mathrm{alb}}\right.$ $=0.95$ ) could be confidently detected by current methods, the minimum value of $P a$ that might be resolved is $1.5 \times 10^{-5} \mathrm{~cm} / \mathrm{s}$ (assuming Qo $=7.5 \mathrm{nl} / \mathrm{min}, \mathrm{D}=20 \mu \mathrm{m}$ and $\mathrm{L}=660 \mu \mathrm{m}$ ). It is likely that Pa for the DVR and AVR is less than this or that the interstitium represents a greater barrier to diffusion of ${ }^{125} \mathrm{I}-\mathrm{al}-$ bumin than the capillary wall. Given that the extent to which perfusion rate can be decreased is technically limited, these experiments demonstrate that free-flow microperfusion might be an inadequate method to measure diffusive albumin efflux in vivo.

Convective transport (solvent drag) of albumin. Methods for inducing a high rate of transcapillary volume flux in the AVR have been established (14) and exploited to examine molecular sieving of albumin. Collectate-to-perfusate ratios of a high molecular weight $\left(2 \times 10^{6}\right)$ fluoresceinated dextran and ${ }^{125}$ I-albumin were compared (Fig. 3 ). Sensitivity of the method

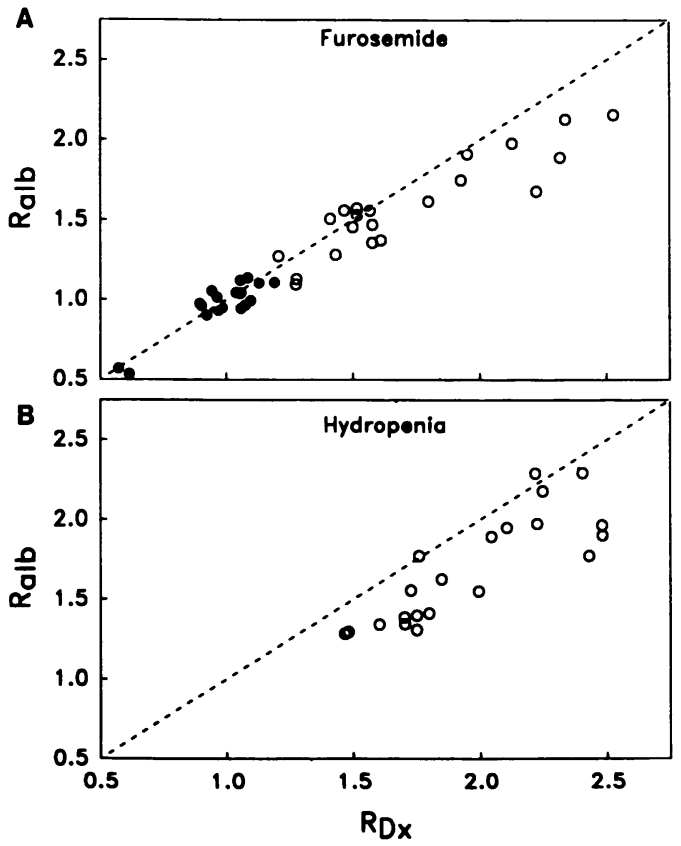

Figure 3. (A) Experimental results of AVR perfusions performed during furosemide diuresis. The collectate-to-perfusate albumin ratio $\left(R_{a l b}\right)$ is shown as a function of $R_{D x}$ for perfusions performed with $5.0 \mathrm{~g} / \mathrm{dl}$ albumin (filled circles, group $0 \mathrm{Jv}-\mathrm{FD} / \mathrm{A}$ ) and $0.1 \mathrm{~g} / \mathrm{dl}$ albumin (open circles, group HJv-FD/A). The dashed line is the line of identity. $(B)$ Experimental results are shown for AVR perfusions performed with $0.1 \mathrm{~g} / \mathrm{dl}$ albumin in hydropenic rats (open circles, group HJv-HD/A). 


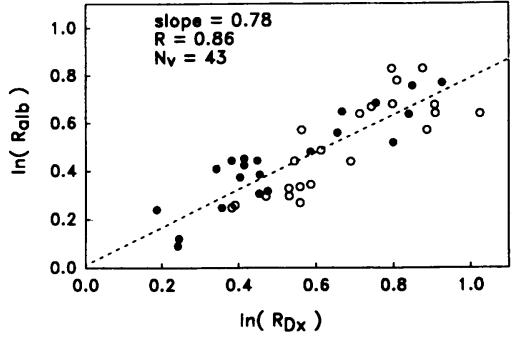

Figure 4. The natural logarithm of $R_{\text {alb }}$ is plotted as a function of the natural logarithm of $R_{D x}$ for a total of 43 high volume flux AVR perfusions performed either during furosemide diuresis (filled circles) or hydropenia (open circles). Accord-

ing to Eq. 9, the slope of the dashed regression line is an estimate of $\sigma_{\mathrm{a}}$ for the AVR wall.

was increased by measuring both markers in the same capillary and identically pipetted samples of perfusate and collectate. A highly significant difference in the collectate-to-perfusate ratios of ${ }^{125}$ I-albumin and FITC-Dx was observed in rats subjected to furosemide diuresis (HJv-FD/A) or maintained in a hydropenic state (HJv-HD/A, Table II and Fig. 2). From these data, the reflection coefficient of the AVR wall to albumin $\left(\sigma_{\mathrm{a}}\right)$ was estimated to be 0.78 (Fig. 4).

Validity of this analysis requires $\beta>3$ (Eqs. 4-7). Assuming an AVR diameter of $20 \mu \mathrm{m}(20)$, Jv for the $\mathrm{HJv}$-FD/A and HJv-HD/A groups was $13.4 \times 10^{-5} \pm 1.4 \times 10^{-5}$ and 14.9 $\times 10^{-5} \pm 0.6 \times 10^{-5} \mathrm{~cm} / \mathrm{s}$, respectively. To satisfy $\beta>3$ with $\sigma_{\mathrm{a}}$ $=0.78, \mathrm{~Pa}$ must be less than $1 \times 10^{-5} \mathrm{~cm} / \mathrm{s}$. The analysis provided above coupled with measurements of albumin permeability in other capillary beds $\left(\mathrm{Pa}<10^{-7} \mathrm{~cm} / \mathrm{s}\right)(21)$ suggests that this criterion is very likely to be fulfilled.

In the DVR, presumably due to its low hydraulic conductivity (13), a high rate of volume flux was not be achieved (group HJv-FD/D, Table II). In view of this, it is not surprising that convective albumin efflux across the DVR wall could not be measured (Table II, Group HJv-FD/D). Based on ultrastructural differences between their endothelia, it seems likely that the DVR reflection coefficient to albumin is higher than that for the AVR. Due to inherent insensitivity of current methods, however, the results in Table II cannot be taken to imply that the DVR wall has a reflection coefficient to albumin of unity.

Mechanism of transcapillary equilibration of albumin in vivo. In the hydropenic Munich-Wistar rat, under experimental conditions in which the ureter has been excised to access the papilla for micropuncture, it has been observed that plasma protein concentration falls during transit from papillary tip to base in the AVR (22-24). Those experiments have been interpreted to demonstrate AVR uptake of water from the papillary interstitium. This is consistent with the need of the microcirculation to reabsorb water deposited by the loops of Henle and collecting tubule (22-24). Perfusion rates in these experiments were similar to blood flow rates in vivo and diffusion of albumin was found to be undetectable. These experiments therefore corroborate that the fall in AVR plasma protein concentration observed in vivo is likely due to water uptake rather than significant albumin efflux.

It also follows that if convection is the dominant mode of albumin transport across the AVR wall during hydropenia, AVR will remove albumin from the papillary interstitium rather than deposit it there. Thus high concentrations of albumin in the medullary interstitium observed in vivo may originate from descending vasa recta or the sparce capillary plexus interposed between DVR and AVR. This is consistent with current data concerning the DVR because, given the absence of lymphatics in the inner medulla, the rates of transport responsible for interstitial albumin accumulation might very well be too low to measure by present methods. A detailed mathematical simulation of such a convective albumin exchanger would be required to accurately define its function.

\section{Acknowledgments}

This work was supported by National Institutes of Health grant R29 DK42495 and a Bioengineering Research Grant from the Whitaker Foundation.

\section{References}

1. Lassen, N. A., J. B. Longley, and L. S. Lilienfield. 1958. Concentration of albumin in renal papilla. Science (Wash. DC). 128:720-721.

2. Slotkoff, L. M., and L. S. Lilienfield. 1967. Extravascular renal albumin. Am. J. Physiol. 212:400-406.

3. Pinter, G. G. 1967. Distribution of chylomicrons and albumin in dog kidney. J. Physiol. (Camb.). 192:761-772.

4. Moffat, D. B. 1969. Extravascular protein in the renal medulla. Q. J. Exp. Physiol. 54:60-67.

5. Pomerantz, R. M., L. M. Slotkoff, and L. S. Lilienfield. 1965. Histochemical and microanatomical differences between renal cortical and medullary interstitium. In Progress in Pyelonephritis. E. H. Kass, editor. F. A. Davis Co., Philadelphia, PA. p. 434.

6. Wilde, W. S., and C. Vorburger. 1967. Albumin multiplier in kidney vasa recta analyzed by microspectrophotometry of T-1824. Am. J. Physiol. 213:12331243.

7. Shimamura, T., and A. B. Morrison. 1973. Vascular permeability of the renal medullary vessels in the mouse and rat. Am. J. Pathol. 71:155-166.

8. Venkatachalam, M. A., and M. J. Karnovsky. 1972. Extravascular protein in the kidney. Lab. Invest. 27:435-444.

9. Bell, R. D., M. J. Keyl, R. F. Schrader, E. W. Jones, and L. P. Henry. 1968. Renal lymphatics: the internal distribution. Nephron. 5:454-463.

10. Kriz, W. J., J. M. Barrett, and S. Peter. 1976. The renal vasculature: anatomical-functional aspects. In Kidney and Urinary Tract Physiology II. Vol. 11. K. Thurau, editor. University Park Press, Baltimore, MD. 1-21.

11. Schwartz, M. M., M. J. Karnovsky, and M. A. Venkatachalam. 1976. Ultrastructural differences between rat inner medullary descending and ascending vasa recta. Lab. Invest. 35:161-170.

12. Pallone, T. L., Y. Yagil, and R. L. Jamison. 1989. Effect of small-solute gradients on transcapillary fluid movement in renal inner medulla. Am. J. Physiol. 257(Renal Fluid Electrolyte Physiol. 26):F547-F553.

13. Pallone, T. L., J. Work, and R. L. Jamison. 1990. The resistance of the descending vasa recta to the transport of water. Am. J. Physiol. 259(Renal Fluid Electrolyte Physiol. 28):F688-F697.

14. Pallone, T. L. 1991. Resistance of ascending vasa recta to transport of water. Am. J. Physiol. 260 (Renal Fluid Electrolyte Physiol. 29):F303-F310.

15. Pallone, T. L. 1991. Transport of $\mathrm{NaCl}$ and water in ascending vasa recta. Am. J. Physiol. 261 (Renal Fluid Electrolyte Physiol. 30):F519-F525.

16. Lowry, O. H., N. J. Rosenbrough, A. L. Farr, and R. J. Randall. 1951. Protein measurement with the Folin phenol reagent. J. Biol. Chem. 193:265275.

17. Patlak, C. S., D. A. Goldstein, and J. F. Hoffman. 1963. The flow of solute and solvent across a two-membrane system. J. Theor. Biol. 5:426-442.

18. Nugent, L. J., and R. K. Jain. 1984. Plasma pharmacokinetics and interstitial diffusion of macromolecules in a capillary bed. Am. J. Physiol. (Heart Circ. Physiol. 15):H129-H137.

19. Friedman, J. J., and S. Witte. 1986. The radial protein concentration profile in the interstitial space of the rat ileal mesentery. Microvasc. Res. 31:277287.

20. Holliger, C., K. V. Lemley, S. L. Schmitt, F. C. Thomas, C. R. Robertson, and R. L. Jamison. 1983. Direct determination of vasa recta blood flow in the rat renal papilla. Circ. Res. 53:401-413.

21. Renkin, E. M. 1977. Multiple pathways of capillary permeability. Circ. Res. 41:735-743.

22. Sanjana, V. M., P. A. Johnston, C. R. Robertson, and R. L. Jamison. 1976. An examination of the transcapillary water flux in the renal medulla. Am.J. Physiol. 231:313-318.

23. Sanjana, V. M., P. A. Johnston, J. L. Troy, W. M. Deen, C. R. Robertson, B. M. Brenner, and R. L. Jamison. 1975. Hydraulic and oncotic pressure measurements in the inner medulla of the mammalian kidney. Am. J. Physiol. 228:19211926.

24. Zimmerhackl, B., C. R. Robertson, and R. L. Jamison. 1985. Fluid uptake in the renal papilla by vasa recta estimated by two methods simultaneously. $A m$. J. Physiol. 248(Renal Fluid Electrolyte Physiol. 17):F347-F353. 